\title{
Changes in TLR-4 expression level and CD14+CD16+ monocyte ratio in the peripheral blood of patients with early diabetic nephropathies
}

\author{
Yu Chen ${ }^{\mathrm{a}, *}$, Kun Men ${ }^{\mathrm{b}}$, Chun-mei Meng ${ }^{\mathrm{a}}$, Jing Ma ${ }^{\mathrm{a}}$, Jian-chao Guo ${ }^{\mathrm{a}}$ \\ a Department of Endocrinology, The Second Hospital of Tianjin Medical University, Tianjin 300211 China \\ b Department of Clinical Laboratory, The Second Hospital of Tianjin Medical University, Tianjin, China
}

*Corresponding author, e-mail: yu_chen_666@126.com

Received 16 May 2019

Accepted 17 Mar 2020

\begin{abstract}
Cytokine-mediated activation of chronic microinflammatory and nonspecific immune responses plays an important role in the progression of early diabetic nephropathy (EDN). The purpose of this study was to investigate Tolllike receptor 4 (TLR-4) levels in monocytes and the CD14+CD16+ monocyte ratios in peripheral blood from diabetic patients with EDN. One hundred and eighty-eight patients with type 2 diabetes mellitus were recruited and divided into 3 groups according to their microalbumin (mALB) content: (1) A-group ( $\mathrm{mALB}<30 \mathrm{mg} / 24 \mathrm{~h}, \mathrm{n}=60$ ); (2) B-group (mALB 30-300 mg/24 h, $\mathrm{n}=64$ ), which was further divided into the losartan potassium treatment group (B-T-group) and the untreated group (B-NT-group); and (3) C-group (mALB $>300 \mathrm{mg} / 24 \mathrm{~h}, \mathrm{n}=64$ ). Additionally, samples from 50 healthy patients were collected as the control group (N-group). Immune turbidimetric assays were used to measure high-sensitivity C-reactive protein (hsCRP) levels in serum. The levels of interleukin 6 (IL-6) in serum were detected by ELISA. CD14+CD16+ monocyte ratios and TLR-4 levels in monocytes were assessed by flow cytometry. There was no significant difference in the levels of hsCRP, IL-6, and TLR-4 and the CD14+CD16+ monocyte ratios between the A-group and N-group. The hsCRP, IL-6, TLR-4 and CD14+CD16+ monocyte levels in the B-group and C-group were notably higher than those in the A-group or N-group. Furthermore, compared with levels in the B-NT-group, TLR-4 and CD14+CD16+ monocyte levels were significantly decreased in the B-T-group. TLR-4 and CD14+CD16+ monocyte levels were increased in patients with EDN and restored with losartan potassium treatment, and the monocyte levels were positively correlated with the decrease in mALB. The TLR-4 level in monocyte and CD14+CD16+ monocyte ratio can be used as new targets for the early diagnosis of clinical diabetic nephropathy.
\end{abstract}

KEYWORDS: early diabetic nephropathy, TLR-4, CD14+CD16+, chronic immune microinflammation

\section{INTRODUCTION}

Type 2 diabetes (T2DM) is a chronic metabolic disease characterized by hyperglycemia, insulin resistance and a relative lack of insulin [1]. Type 2 diabetes is caused by complex genetic-environment interactions as well as other risk factors such as obesity and a sedentary lifestyle [2,3]. T2DM and its complications, such as cardiovascular disease, diabetic neuropathy and nephropathy, seriously threaten human life and health [4-7]. With the incidence of increasing T2DM, finding an ideal treatment has become one of the primary tasks in combating this disease. Diabetic nephropathy is one of the most common and serious microvascular complications in diabetic patients [8].

Inflammation is associated with insulin resistance, diabetes and diabetes-related complica- tions $[9,10]$. Monocytes and Toll-like receptor (TLR) family are associated with inflammatory responses [11,12]. Monocytes in the peripheral blood play an important role in innate immunity and response to infection. Monocytes include 3 subtypes: CD14+CD16-; CD14+CD16+; and CD14-CD16+ [13]. In healthy people, 90$95 \%$ of typical monocytes have high expression of CD14 (CD14+) and low expression of CD16 (CD16-). However, inflammation and infection lead to co-expression of CD14 and CD16 (CD14+CD16+) on the surface of monocytes [14]. CD14+CD16+ monocytes secrete high levels of TNF- $\alpha$ and low levels of anti-inflammatory factors such as interleukin-10 (IL-10). In addition, the TLR family is also very important for innate immunity [15]. TLR-4 is associated with atherosclerosis, diet-induced obesity and obesity associated insulin 
resistance [16]. Previous studies have shown that monocytes and TLRs in diabetic patients have a proinflammatory effect [10]. However, the correlation between TLR-4 level in monocytes and the CD14+CD16+ monocyte ratio in peripheral blood from diabetic patients with EDN has been rarely reported.

In this study, the levels of TLR-4 and the CD14+CD16+ monocyte ratios in EDN patients were investigated by immunoturbidimetry, ELISA and flow cytometry. The results of this study demonstrate that the TLR-4 level in monocyte and CD14+CD16+ monocyte ratio can be used as new targets for the early diagnosis of clinical diabetic nephropathy.

\section{MATERIALS AND METHODS}

\section{Patients}

One hundred and eighty-eight patients with T2DM (from January to December 2015, Department of Endocrinology, Second Hospital of Tianjin Medical University) were recruited for this study. Inclusion criteria are as follows: pathologically confirmed T2DM with EDN; there was no use of glucocorticoids, anti-infection and anti-inflammatory drugs during the first three months of treatment. This study was subject to approval by the ethics committee of the Second Hospital of Tianjin Medical University and conducted in accordance with the provisions of the Declaration of Helsinki, Good Clinical Practice guidelines and local laws and regulations. All participants provided written informed consent and confirmed their willingness to perform glucose self-monitoring. Exclusion criteria are as follows: patients with disease of the brain, stomach, lung or other important organs; patients with hematological diseases; and patients with allergies to this treatment drug. The T2DM patients were divided into 3 groups according to their microalbumin (mALB) content: (1) A-group (mALB < $30 \mathrm{mg} / 24 \mathrm{~h}, \mathrm{n}=60$ ); (2) B-group (mALB $30-300 \mathrm{mg} / 24 \mathrm{~h}, \mathrm{n}=64$ ), which was further divided into the losartan potassium treatment group (B-Tgroup, $\mathrm{n}=32$, oral losartan potassium $50 \mathrm{mg}$ once a day) and the untreated group (B-NT-group, $n=32$ ); and (3) C-group (mALB $>300 \mathrm{mg} / 24 \mathrm{~h}, \mathrm{n}=64$ ). Losartan potassium (H20080371) was produced by the Yangtze River Pharmaceutical Group (Jiangsu, China). Patients in the losartan potassium treatment group took the losartan potassium according to the drug instructions. Additionally, 50 healthy participants were recruited (N-group), and all healthy participants met the inclusion and exclusion criteria of this study. All volunteers were observed and monitored for one year. The characteristics of the study participants are shown in Table 1.

\section{Detection of general biochemical indexes in patients}

Fasting blood glucose (FBG), total cholesterol (TC), mALB and serum creatinine (Scr) levels in patients were measured using a BS2000M automatic biochemical analyzer (Mindray, Shenzhen, China), and Hemoglobin A1c (HbA1c) level was detected using a D-10 glycosylated hemoglobin analyzer (Bio-Rad, California, USA) according to the manufacturer's instructions.

\section{Immunoturbidimetric assay}

The level of hsCRP in serum was assessed by immunoturbidimetric assay. A C-reactive protein assay kit-CRP (immunoturbidimetry) (Leadman, Beijing, China) and an IMMAGE 800 specific protein analyzer (Beckman Coulter, Inc., California, USA) were used in this study.

\section{Enzyme-linked immunosorbent assay (ELISA)}

Levels of IL- 6 in serum were measured by ELISA. Human IL-6 ELISA kit was purchased from Abcam (Cambridge, USA) and used according to the manufacturer's protocol. Serum was collected by centrifugation at $2000 \times \mathrm{g}$ for $10 \mathrm{~min}$ and diluted with Sample Diluent NS. The serum was seeded into 96-well plate $(50 \mu \mathrm{l} /$ well), then incubated with antibody ( $50 \mu \mathrm{l} /$ well) for $1 \mathrm{~h}$ at room temperature on a plate shaker set to $400 \mathrm{rpm}$. Then, the plates were washed 3 times with PBST (containing 0.05\% Tween-20). TMB Substrate $(100 \mu l)$ was added to each well and incubated for $10 \mathrm{~min}$ in the dark on a plate shaker set to $400 \mathrm{rpm}$. Then, $100 \mu 1$ of stop solution was added to each well, and the absorbance value of each well was measured at $450 \mathrm{~nm}$ with the Thermo Fisher Multiskan FC (Thermo Fischer Scientific, Waltham, MA, USA). All samples were measured in duplicate.

\section{Isolation of peripheral blood mononuclear cells (PBMCs) and monocytes}

Peripheral blood was collected from patients and diluted at 1:1 using Hanks buffer. The diluted peripheral blood was slowly added into a centrifuge tube containing lymphocyte separation fluid (Solarbio, Beijing, China). The volume ratio of the diluted peripheral blood to lymphocyte separation solution was $2: 1$. The cells were centrifuged horizontally at 
Table 1 The characteristics of the study participants.

\begin{tabular}{lccccc}
\hline Characteristic & A-group & B-T-group & B-NT-group & C-group & N-group \\
\hline Number & 60 & 32 & 32 & 64 & 50 \\
Sex (M/F) & $28 / 32$ & $13 / 19$ & $17 / 15$ & $35 / 29$ & $24 / 26$ \\
Age (year)* & $53(48-58)$ & $59(47-65)$ & $56(45-71)$ & $61(51-70)$ & $49(44-62)$ \\
Disease duration (month) $^{*}$ & $84(60-96)$ & $90(70-108)$ & $72(59-84)$ & $88(54-98)$ & \\
\hline
\end{tabular}

* Data are expressed as median (inter quartile range); M, male; F, female.

$250 \times g$ for $20 \mathrm{~min}$ at room temperature. A capillary pipette was inserted into the white cell layer to aspirate the cells, and the cells were transferred to another tube. Then, these cells were resuspended in Hanks buffer. The magnetic separation method was used for monocyte isolation as previously described [17].

\section{Flow cytometry assay}

Monocytes $\left(1 \times 10^{5}\right.$ cells $)$ were washed with PBS buffer (containing 1\% fetal bovine serum) and centrifuged at $250 \times g$ for $15 \mathrm{~min}$. Then, the cells were resuspended in PBS buffer and fixed with $4 \%$ formaldehyde for $15 \mathrm{~min}$ at room temperature. The cells were permeabilized with $100 \%$ and $90 \%$ methanol, and then incubated on ice for $30 \mathrm{~min}$. Five microliters of primary antibodies, anti-CD16 (Becton, Dickinson and Company, New Jersey, USA), anti-CD14 (Becton-Dickinson) and anti-TLR-4 (Becton-Dickinson) were added into monocyte suspension sample $(100 \mu \mathrm{l})$ at $4^{\circ} \mathrm{C}$ and incubated for $30 \mathrm{~min}$ in the dark. Cells were centrifuged at $250 \times g$ for $20 \mathrm{~min}$ and washed with PBS buffer. Then, the cells were resuspended in PBS buffer. Then, the samples were analyzed using a Coulter XL flow cytometer (Beckman Coulter).

\section{Statistical analysis}

SPSS22.0 (version 22.0; IBM Corp., Armonk NY, USA) was used for statistical analysis. All data are expressed as the mean \pm standard deviation (SD). Analysis of variance between groups was performed for variance analysis. Student's $t$-test was applied to compare 2 groups. one-way analysis of variance was applied to compare all pairs of the group. Pearson's correlation coefficients were calculated to analyze the correlation of hsCRP, IL- 6 and CD14+CD16+ monocyte levels. In order to estimate the accuracy of the EDN prediction, receiver operating characteristics (ROC) curve was made, and areas under the curve (AUC) was calculated. $p<0.05$ was considered statistically significant.

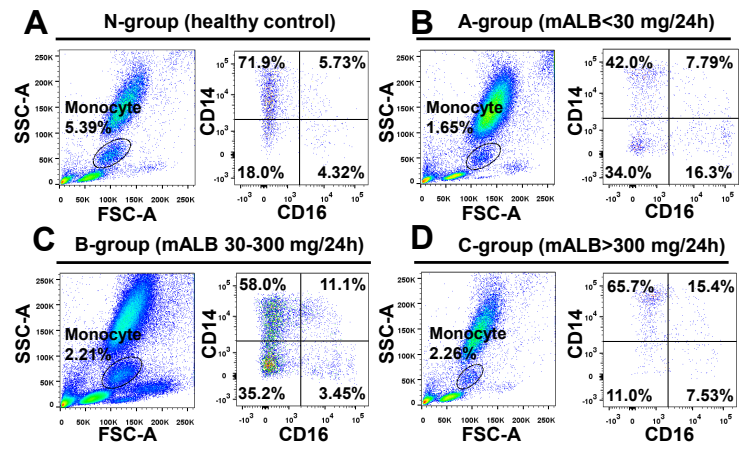

Fig. 1 Flow cytometry analysis of CD14+CD16+ monocyte ratio of: (A) a healthy patient in N-group, (B) a T2DM patient in A-group, (C) a T2DM patient in B-group, and (D) a T2DM patient in C-group.

\section{RESULTS}

\section{Blood pressure and general biochemical indexes}

The morning blood pressures of the patients in each group were within the range of 120-140/70$90 \mathrm{mmHg}$. In addition, there were no significant differences in the levels of FBG, TC, HbA1c and Scr among the A-group, B-group and C-group (Table 2, $p>0.05)$.

\section{The levels of hsCRP, IL-6, TLR-4 and CD14+ CD16+ monocytes in the A, B, C and N groups}

To investigate TLR-4 levels in monocytes and the CD14+CD16+ monocyte ratios in peripheral blood from patients with T2DM, 188 patients with T2DM were recruited and divided into 3 groups according to their microalbumin (mALB) content: (1) Agroup (mALB < $30 \mathrm{mg} / 24 \mathrm{~h}, \mathrm{n}=60$ ); (2) $\mathrm{B}$-group (mALB 30-300 mg/24 h, $\mathrm{n}=64$ ); and (3) C-group (mALB > $300 \mathrm{mg} / 24 \mathrm{~h}, \mathrm{n}=64$ ). The normality test showed that the hsCRP, IL-6 and TLR-4 levels in monocytes and $\mathrm{CD} 14+\mathrm{CD} 16+$ monocyte ratios in each group were normally distributed (Fig. 1, Table 3). There were no significant differences in hsCRP, IL-6 and TLR-4 levels in monocytes and 
Table 2 General biochemical indexes of patients.

\begin{tabular}{lcccccc}
\hline Group & $\mathrm{n}$ & $\begin{array}{c}\mathrm{FBG} \\
(\mathrm{mmol} / \mathrm{l})\end{array}$ & $\begin{array}{c}\mathrm{TC} \\
(\mathrm{mmol} / \mathrm{l})\end{array}$ & $\begin{array}{c}\mathrm{mALB} \\
(\mathrm{mg} / 24 \mathrm{~h})\end{array}$ & $\begin{array}{c}\text { HbA1c } \\
(\%)\end{array}$ & $\begin{array}{c}\text { Scr } \\
(\mu \mathrm{mol} / \mathrm{l})\end{array}$ \\
\hline $\mathrm{N}$ & 50 & $4.80 \pm 0.40$ & $4.90 \pm 0.99$ & $5.51 \pm 0.45$ & $4.71 \pm 0.50$ & $67.3 \pm 4.51$ \\
$\mathrm{~A}$ & 60 & $8.94 \pm 2.45$ & $4.97 \pm 1.17$ & $6.71 \pm 0.71$ & $8.66 \pm 1.89$ & $70.4 \pm 8.41$ \\
$\mathrm{~B}$ & 64 & $9.17 \pm 2.31$ & $4.54 \pm 1.52$ & $55.63 \pm 6.93$ & $8.87 \pm 2.29$ & $72.5 \pm 9.34$ \\
$\mathrm{C}$ & 64 & $10.17 \pm 3.19$ & $5.09 \pm 1.26$ & $552.94 \pm 96.33$ & $9.11 \pm 1.65$ & $75.9 \pm 9.76$ \\
\hline
\end{tabular}

Table 3 The levels of hsCRP, IL-6, TLR-4 and CD14+CD16+ monocyte in A, B, C and N groups.

\begin{tabular}{lccccc}
\hline Group & $\mathrm{n}$ & $\begin{array}{c}\text { hsCRP } \\
(\mathrm{mg} / \mathrm{l})\end{array}$ & $\begin{array}{c}\mathrm{IL}-6 \\
(\mathrm{pg} / \mathrm{ml})\end{array}$ & $\begin{array}{c}\text { TLR-4 } \\
(\%)\end{array}$ & $\begin{array}{c}\text { CD14+CD16+ } \\
(\%)\end{array}$ \\
\hline $\mathrm{N}$ & 50 & $0.791 \pm 0.306$ & $10.787 \pm 3.264$ & $11.024 \pm 3.604$ & $6.10 \pm 2.591$ \\
$\mathrm{~A}$ & 60 & $1.015 \pm 0.408$ & $13.312 \pm 4.246$ & $11.341 \pm 4.095$ & $7.35 \pm 3.736$ \\
$\mathrm{~B}$ & 64 & $2.279 \pm 0.503^{*}$ & $23.456 \pm 4.285^{*}$ & $17.531 \pm 5.385^{*}$ & $11.5 \pm 4.550^{*}$ \\
$\mathrm{C}$ & 64 & $3.217 \pm 0.967^{*}$ & $32.317 \pm 8.672^{*}$ & $19.961 \pm 5.231^{*}$ & $15.6 \pm 4.807^{*}$ \\
\hline
\end{tabular}

$* p<0.05$ versus the $\mathrm{N}$ group.

Table 4 IL-6, TLR-4 and mALB levels and CD14+CD16+ monocyte ratios in B-T-group and B-NT-group.

\begin{tabular}{lcr}
\hline Index & $\begin{array}{c}\text { B-T-group } \\
(\mathrm{n}=32)\end{array}$ & $\begin{array}{c}\text { B-NT-group } \\
(\mathrm{n}=32)\end{array}$ \\
\hline IL-6 (pg/ml) & $20.33 \pm 3.66$ & $22.04 \pm 3.86$ \\
TLR-4 (\%) & $14.78 \pm 5.01^{*}$ & $19.81 \pm 2.57$ \\
CD14+CD16+ (\%) & $5.94 \pm 2.04^{*}$ & $9.49 \pm 3.15$ \\
mALB (mg/24 h) & $20.61 \pm 3.21^{*}$ & $68.37 \pm 5.26$ \\
\hline
\end{tabular}

$* p<0.05$ versus B-NT-group.

CD14+CD16+ monocyte ratio between the A-group and N-group ( $p>0.05$, Fig. 1, Table 3). However, the levels of hsCRP, IL- 6 and TLR-4 in monocytes and $\mathrm{CD} 14+\mathrm{CD} 16+$ monocyte ratios in the B-group and C-group were notably higher than those in the N-group ( $p<0.05$, Fig. 1, Table 3 ). We also performed a one-way analysis of variance, and the results show that the hsCRP, IL- 6 and TLR-4 levels in monocytes and $\mathrm{CD} 14+\mathrm{CD} 16+$ monocyte ratios have a significant effect on EDN progress $(p<0.05$, Table 3).

\section{The levels of hsCRP, IL-6, TLR-4 and CD14+CD16+ monocytes in the B-T-group and B-NT-group}

Sixty-four patients in the B-group (mALB 30$300 \mathrm{mg} / 24 \mathrm{~h}$ ) were divided into 2 groups with 32 cases in each group: the losartan potassium treatment group (B-T-group, oral losartan potassium $50 \mathrm{mg}$ once per day) and the untreated group (B-NT-group). The levels of IL- 6 and TLR-4 in monocytes, mALB and CD14+CD16+ monocyte ra-
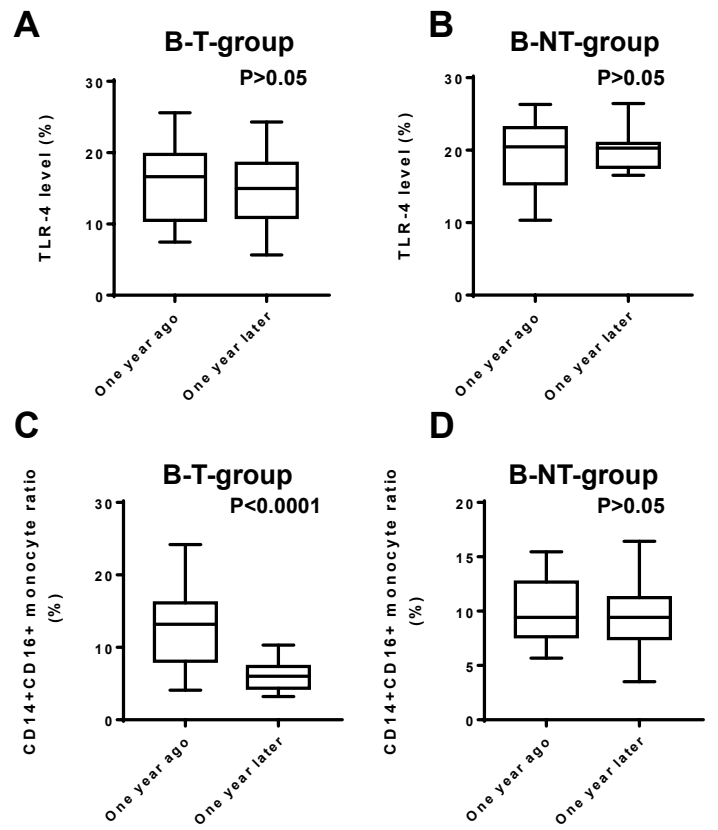

Fig. 2 Time-dependent analysis of TLR-4 and CD14+ CD16+ monocytes in: $(A, B)$ the B-T-group and $(C, D)$ the B-NT-group.

tios were retested after one year. As shown in Table 4, the levels of TLR-4 in monocytes, mALB and CD14+CD16+ monocyte ratios in the B-T-group were significantly higher than those in the B-NTgroup $(p<0.05)$. However, the IL-6 level was not significantly different between the B-T-group and the B-NT-group ( $p>0.05$, Table 4$)$. At the same 

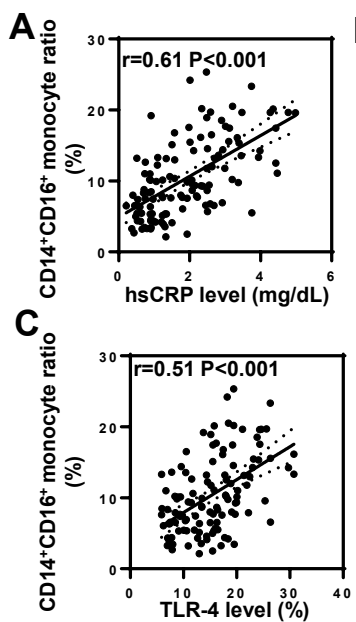

E

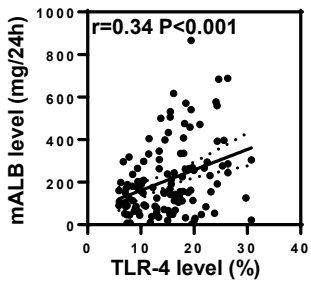

Fig. 3 Pearson's correlation scatter plot of CD14+CD16+ monocyte ratio with (A) hsCRP levels, (B) IL-6 levels, and (C) TLR-4 levels. Pearson's correlation scatter plot of mALB levels with (D) CD14+CD16+ monocyte ratio and (E) TLR-4 levels.

time, we also compared the data of group B one year ago and one year later. However, due to the short follow-up ime, we only observed a significant decrease in CD14+CD16+ monocyte ratios in B-Tgroup (Fig. 2).

\section{Correlation between hsCRP, IL-6 and TLR-4 levels in monocytes and CD14+CD16+ monocyte ratio}

Finally, we analyzed the correlation of hsCRP, IL-6 and TLR-4 in monocytes and CD14+CD16+ monocyte levels in $188 \mathrm{~T} 2 \mathrm{DM}$ patients. As shown in Fig. 3(A-C), the expression levels of hsCRP, IL-6 and TLR-4 in monocytes were positively correlated with CD14+CD16+ monocyte ratios. In addition, we also compared the correlation between TLR-4 in monocytes and CD14+CD16+ monocyte ratios to mALB levels. The results showed a significant positive correlation between TLR-4 in monocytes and CD14+CD16+ monocyte ratios and mALB levels (Fig. 3DE).
A

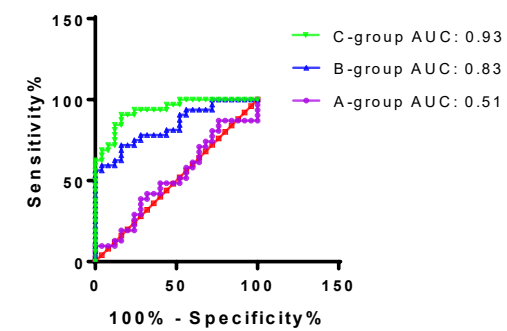

B

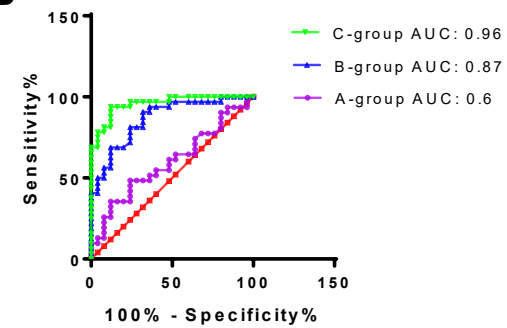

Fig. 4 The receiver operating characteristics (ROC) curves of the levels of TLR-4 in (A) monocytes and (B) CD14+CD16+ monocyte ratios for EDN diagnosis.

The ROC curves of the levels of TLR- 4 in monocytes and CD14+CD16+ monocyte ratios for EDN diagnosis

To confirm that the levels of TLR-4 in monocytes and CD14+CD16+ monocyte ratios had high efficacy in predicting EDN, mALB level-dependent ROC analyses were conducted. The A-, B- and C-group AUC values of TLR-4 levels in monocytes for the prediction of EDN were 0.51, 0.83 and 0.93, respectively (Fig. 4a). In addition, the A-, B- and C-group AUC values of CD14+CD16+ monocyte ratios for the prediction of EDN were 0.6, 0.87 and 0.96, respectively (Fig. 4b). The results show that the levels of TLR-4 in monocytes and CD14+CD16+ monocyte ratios can be used as sensitive indicators for EDN diagnosis in patients with mALB level greater than $30 \mathrm{mg} / 24 \mathrm{~h}$.

\section{DISCUSSION}

The pathogenesis of EDN is very complex and has not yet been fully elucidated, but many studies have pointed out that the inflammatory pathway is the central link in the progression of EDN, and the extracellular matrix proliferation caused by continuous inflammation promotes the progression of EDN. The research on inflammation in EDN will help develop new therapeutic targets and detection indicators. In this study, TLR-4 levels in mono- 
cytes and CD14+CD16+ monocyte ratios, which can reflect the inflammatory state and proinflammatory functions, were used to explore the role of immune microinflammation in the EDN of T2DM. The results showed that there was no significant difference in the levels of hsCRP, IL- 6 , TLR- 4 and CD14+CD16+ monocytes between the N-group and A-group (mALB $<30 \mathrm{mg} / 24 \mathrm{~h}$ ), indicating that there was no chronic microinflammatory reaction in the early stage of kidney injury in diabetes mellitus. The levels of hsCRP, IL-6, TLR-4 and CD14+CD16+ monocytes in DN microalbuminuria patients (Bgroup, mALB $30-300 \mathrm{mg} / 24 \mathrm{~h}$ ) or DN patients with a large amount of proteinuria (C-group, mALB > $300 \mathrm{mg} / 24 \mathrm{~h}$ ) were significantly higher than those in healthy controls or diabetic patients without renal injury (A-group, $\mathrm{mALB}<30 \mathrm{mg} / 24 \mathrm{~h}$ ). This trend is consistent with reports by Yang et al [18]. However, compared to the research by Yang et al, our research focuses on EDN and provides a larger sample size. The above results indicated that with the increase in mALB, renal injury was gradually aggravated and the level of chronic microinflammation was increased.

Several studies have shown that the expression of TLR-4 in the kidneys of patients with DN is significantly higher than that in normal people, with the proximal tubule epithelial cells being obvious. Ligands filtered from the glomerulus such as high glucose, high-speed surging family B1 protein and glycosylation products can be more quickly recognized by TLR-4. In patients with T2DM, the TLR4 receptor may simultaneously recognize high glucose, high free fatty acids and glycosylation products to regulate the immune inflammatory response and promote the development of DN [19]. In addition, Brooks-Worrell et al [20] found that the proportion of CD14+CD16+ monocytes in peripheral blood was elevated in autoantibody-negative diabetic patients with ketosis and could further differentiate into their corresponding subtypes. In this study, we are demonstrating that TLR-4 levels in monocytes and CD14+CD16+ monocyte ratios have a significant effect on EDN progress. However, its specific mechanism still needs further research.

Losartan potassium is a class of angiotensin receptor blocker (ARB) drugs that can reduce urinary protein and inhibit inflammation [21]. Losartan potassium can selectively and competitively bind to angiotensin receptor I subtype (ATI), block ATImediated physiological responses and improve the glomerular hypermetabolism [21]. Studies have also confirmed that ARBs inhibit inflammation by inhibiting the NF-KB pathway [22]. This study also showed a significant decrease in the levels of TLR-4 and CD14+CD16+ monocytes in the B-Tgroup compared to those in the B-NT-group ( $p<$ $0.05)$. These results further confirmed that when the patients were in the microalbuminuria stage of DN, treatment with ARB drugs significantly decreased the levels of TLR-4 and CD14+CD16+ monocytes, decreased chronic microinflammation and the corresponding level of uric microalbumin and played a certain role in alleviating and reversing the early damage in diabetic nephropathy. Moreover, CD14+CD16+ monocytes were positively correlated with the clinical inflammatory markers, hsCRP and IL-6, further indicating their predictive value in DN (including the early stage). However, the IL-6 level was not significantly different after losartan potassium treatment. This result suggests that losartan potassium may not inhibit inflammation through the TLR-4-IL-6 signaling axis. But this still needs further research.

In conclusion, the TLR-4 levels and $\mathrm{CD} 14+\mathrm{CD} 16+$ monocyte ratio were increased in patients with EDN, restored with losartan potassium treatment and were positively correlated with the decrease in MALB level. ROC analyses showed that the TLR-4 level and CD14+CD16+ monocyte ratio can be used as new targets for the early diagnosis of clinical diabetic nephropathy.

\section{REFERENCES}

1. Wu Y, Ding Y, Tanaka Y, Zhang W (2014) Risk factors contributing to type 2 diabetes and recent advances in the treatment and prevention. Int J Med Sci 11, 1185-1200.

2. Ismail-Beigi F (2012) Pathogenesis and glycemic management of type 2 diabetes mellitus: a physiological approach. Arch Iran Med 15, 239-246.

3. Gastaldelli A (2011) Role of beta-cell dysfunction, ectopic fat accumulation and insulin resistance in the pathogenesis of type 2 diabetes mellitus. Diabetes Res Clin Pract 93S1, 60-65.

4. Pan $H$, Huang $H$, Zhang L, Ma S, Yang H, Wang $H$ (2019) Adjusting internal organs and dredging channel electroacupuncture treatment prevents the development of diabetic peripheral neuropathy by downregulating glucose-related protein 78 (GRP78) and caspase-12 in streptozotocin-diabetic rats. $J \mathrm{Di}$ abetes 11, 928-937.

5. Afarideh M, Zaker Esteghamati V, Ganji M, Heidari B, Esteghamati S, Lavasani S, Ahmadi M, Tafakhori A, et al (2019) Associations of serum S100B and $\mathrm{S} 100 \mathrm{P}$ with the presence and classification of diabetic 
peripheral neuropathy in adults with type 2 diabetes: A case-cohort study. Can J Diabetes 43, 336-344.

6. Archundia Herrera MC, Subhan FB, Chan CB (2017) Dietary patterns and cardiovascular disease risk in people with type 2 diabetes. Curr Obes Rep 6, 405-413.

7. Koc AS, Sumbul HE (2018) Renal cortical stiffness obtained by shear wave elastography imaging is increased in patients with type 2 diabetes mellitus without diabetic nephropathy. $J$ Ultrasound 21, 279-285.

8. Vigersky RA (2011) An overview of management issues in adult patients with type 2 diabetes mellitus. J Diabetes Sci Technol 5, 245-250.

9. Taha IM, Abdu Allah AM, Abd El Gayed EM (2018) Expression of toll-like receptor 4 and its connection with type 2 diabetes mellitus. Cell Mol Biol 64, 15-20.

10. Ren X, Mou W, Su C, Chen X, Zhang H, Cao B, Li X, Wu D, et al (2017) Increase in peripheral blood intermediate monocytes is associated with the development of recent-onset type 1 diabetes mellitus in children. Int J Biol Sci 13, 209-218.

11. Shi C, Pamer EG (2011) Monocyte recruitment during infection and inflammation. Nat Rev Immunol 11, 762-774.

12. Ullah MO, Sweet MJ, Mansell A, Kellie S, Kobe B (2016) TRIF-dependent TLR signaling, its functions in host defense and inflammation, and its potential as a therapeutic target. J Leukoc Biol 100, 27-45.

13. Sugimoto C, Hasegawa A, Saito Y, Fukuyo Y, Chiu KB, Cai Y, Breed MW, Mori K, et al (2015) Differentiation kinetics of blood monocytes and dendritic cells in macaques: insights to understanding human myeloid cell development. J Immunol 195, 1774-1781.

14. Ziegler-Heitbrock L (2007) The CD14+CD16+ blood monocytes: their role in infection and inflammation. J Leukoc Biol 81, 584-592.

15. Ermis Karaali Z, Candan G, Aktuglu MB, Velet M,
Ergen A (2019) Toll-like receptor 2 (TLR-2) gene polymorphisms in type 2 diabetes mellitus. Cell $J \mathbf{2 0}$, 559-563.

16. Ghanim H, Mohanty P, Deopurkar R, Sia CL, Korzeniewski K, Abuaysheh S, Chaudhuri A, Dandona P (2008) Acute modulation of toll-like receptors by insulin. Diabetes Care 31, 1827-1831.

17. Flo RW, Naess A, Lund-Johansen F, Maehle BO, Sjursen H, Lehmann V, Solberg CO (1991) Negative selection of human monocytes using magnetic particles covered by anti-lymphocyte antibodies. $J$ Immunol Methods 137, 89-94.

18. Yang M, Gan H, Shen Q, Tang W, Du X, Chen D (2012) Proinflammatory CD14+CD16+ monocytes are associated with microinflammation in patients with type 2 diabetes mellitus and diabetic nephropathy uremia. Inflammation 35, 388-396.

19. Kaur H, Chien A, Jialal I (2012) Hyperglycemia induces toll like receptor 4 expression and activity in mouse mesangial cells: relevance to diabetic nephropathy. Am J Physiol Renal Physiol 303, 1145-1150.

20. Brooks-Worrell BM, Iyer D, Coraza I, Hampe CS, Nalini R, Ozer K, Narla R, Palmer JP, et al (2013) Islet-specific T-cell responses and proinflammatory monocytes define subtypes of autoantibodynegative ketosis-prone diabetes. Diabetes Care 36, 4098-4103.

21. Matavelli LC, Huang J, Siragy HM (2011) Angiotensin AT(2) receptor stimulation inhibits early renal inflammation in renovascular hypertension. Hypertension 57, 308-313.

22. Rompe F, Artuc M, Hallberg A, Alterman M, Stroder K, Thone-Reineke C, Reichenbach A, Schacherl J, et al (2010) Direct angiotensin II type 2 receptor stimulation acts anti-inflammatory through epoxyeicosatrienoic acid and inhibition of nuclear factor $\kappa \mathrm{B}$. Hypertension 55, 924-931. 